\title{
Bacteriophages of Bacillus cereus and of Crystal-forming Insect Pathogens Related to $B$. cereus
}

\author{
By J. R. NORRIS \\ Department of Bacteriology, University of Glasgow
}

(Received 9 March 1961)

SUMMARY

Four phages isolated from bacteria of the Bacillus cereus group are described. They show cross-reactions between crystal-forming and non-crystal-forming strains which are deficient in ability to produce a lecithinase $\mathrm{C}$. The implications of this finding are discussed.

\section{INTRODUCTION}

Hannay's rediscovery of the parasporal body in the insect pathogen Bacillus thuringiensis (Hannay, 1953) and the subsequent identification of this crystalline protein as the toxin of the organism (Angus, 1954) have stimulated considerable interest in crystal-synthesizing aerobic spore-formers and several workers have discussed the relationship between these organisms and $B$. cereus (Toumanoff, 1956; Le Corroller, 1958; Heimpel \& Angus, 1958). Most of the crystal-formers produce an active lecithinase $\mathrm{C}$, as do the majority of strains of $\boldsymbol{B}$. cereus; but two strains, the so-called $\boldsymbol{B}$. entomocidus var. entomocidus and $\boldsymbol{B}$. entomocidus var. subtoxicus of Heimpel \& Angus (1958) are distinguished from the rest by absence of lecithinase activity and by failure to produce acetylmethylcarbinol.

During work with Bacillus entomocidus var. entomocidus phage plaques appeared spontaneously in slope cultures and a study of the phage responsible, and subsequently of others, was undertaken in an attempt to throw some light on the interrelationships of this group of bacteria.

\section{METHODS}

Bacteria. The work involved 21 typical lecithinase-producing strains of Bacillus cereus, 12 lecithinase-producing strains of $B$. cereus var. mycoides and 13 lecithinaseproducing crystal-formers. Two lecithinase-negative crystal-formers, $B$. entomocidus var. entomocidus and B. entomocidus var. subtoxicus were supplied by Dr C. L. Hannay and a further strain, G2, was isolated from larvae of Galleria mellonella. A crystal-forming organism which produced a very small amount of lecithinase $\mathbf{C}$, G1, was isolated from the same stock of larvae at an earlier date. Three lecithinasenegative strains of $B$. cereus (strains 634, 826, 827) were obtained from Dr Ellen Garvie (see Stone, 1952) and had originally been isolated from milk. A lecithinasenegative strain of $\boldsymbol{B}$. cereus var. mycoides, strain A.C., was a laboratory stock culture of uncertain origin.

Lecithinase. Lecithinase production was detected by growth on egg-yolk agar. Most of the organisms studied were active lipase producers and this may lead to 
confusion when egg-yolk agar is used to detect lecithinase activity; for this reason results were checked with the lecithin agar described by Willis (1960).

Acetylmethylcarbinol production. Ability to produce acetylmethylcarbinol was examined by the method described by Smith, Gordon \& Clark (1952).

Production of acid from carbohydrates. Ability to produce acid from carbohydrates was tested in the presence of ammonia- $\mathbf{N}$ on the solid medium of Smith et al. (1952) as used by Heimpel \& Angus (1958) when studying these organisms.

Media. Cultures for the production of phage stocks and for phage sensitivity testing were grown, usually from spore inocula, on $1 \%(w / v)$ nutrient agar plates containing $1 \%(\mathrm{w} / \mathrm{v})$ peptone and $0.5 \%(\mathrm{w} / \mathrm{v}) \mathrm{NaCl}$. Comparison with several other growth media showed that this relatively poor medium gave the most readily visible plaques.

Isolation of phages. Four phages were isolated and used in the investigation. When crude filtrates of Bacillus entomocidus var. entomocidus were spotted on to lawns of the same organism the plaques formed were all clear and indistinguishable from one another, but on lawns of the subtoxicus variety two types of plaque were formed: (i) clear plaques from which were isolated phage $\mathbf{A}$ which gave similar clear plaques on the variety entomocidus; (ii) turbid plaques yielding phage $\mathbf{B}$ which also gave clear plaques on the entomocidus variety.

When 7-day-old broth cultures of Bacillus entomocidus var. subtoxicus are streaked on to agar plates many of the resulting colonies are phantom forms (Nungester, 1929) collapsing after incubation for 2 days to form thin flat transparent 'ghost' colonies. The organisms remain in this form indefinitely on subculture and the effect is probably due to phage although attempts to demonstrate this conclusively have so far failed. When filtrates of broth cultures of a phantom variant of this organism were spotted on to lawns of organism G1, a few discrete phage plaques were formed from which a further phage, phage $\mathrm{C}$, was isolated.

Cultures of the lecithinase-negative Bacillus cereus strain 826 (see Stone, 1952) often showed spontaneously developing phage plaques which yielded the fourth phage, phage $\mathrm{D}$.

Phage A was routinely propagated on Bacillus entomocidus var. entomocidus, phage $\mathbf{B}$ on $\boldsymbol{B}$. entomocidus var. subtoxicus, phage $\mathbf{C}$ on organism $\mathbf{G 1}$ and phage $\mathbf{D}$ on $B$. cereus 826 . The propagating strains were also used as indicator strains when titres of phage preparations were being determined.

Phage sensitivity tests. As mentioned by McCloy (1951) some strains of Bacillus cereus produce colicine-like agents whose effects can simulate confluent phage lysis at low dilutions. This is also true of many crystal-formers and the effects of these agents were readily seen when undiluted filtrates of phage preparations were dropped on to plates inoculated with test organisms. Such non-specific lysis was never seen when phage preparations were diluted $1 / 10$ before use; sensitivity tests were therefore carried out at this dilution. Positive results observed at phage concentrations which gave confluent lysis were always confirmed by the production of separate phage plaques at higher dilutions.

Phage stocks. Phages were purified by picking from single plaques on the routine propagating strain at least four successive times and grown by spreading a few drops of a sterile-filtered preparation on agar plates the surfaces of which were inoculated with cultures of phage-sensitive organisms, and incubating overnight at 
$30^{\circ}$. The resulting growth was harvested into a few $\mathrm{ml}$. of peptone water and the suspension sterilized by passing it through a Hemming centrifugal filter (Beaumaris Instrument Co. Ltd., Rosemary Lane, Beaumaris, Anglesey). Phage preparations containing $10^{10}$ or $10^{11}$ plaque-forming units $/ \mathrm{ml}$. on the corresponding indicator strains were readily prepared by this method which gave, on the whole, higher titres than fluid culture techniques.

Titration of phage. Rough estimates of numbers of phage particles were made by the modified Miles \& Misra technique described by McCloy (1958). Accurate titrations were made by the soft agar-layer method described by Adams (1959). In both methods the plates were inoculated with spore suspensions of the indicator organism produced by harvesting 10-day-old agar slope cultures in sterile distilled water and pasteurizing the resulting suspensions at $75^{\circ}$ for $15 \mathrm{~min}$. Control plates without added phage were always set up as a check against spontaneous phage lysis. With some organisms indicator plates prepared in this way always showed spontaneous phage plaques, but phage was usually absent from $16 \mathrm{hr}$. broth cultures grown from single plaque-free colonies; such cultures were used to inoculate titration plates instead of the usual spore suspensions.

\section{RESULTS}

\section{Acetylmethylcarbinol production}

Acetylmethylcarbinol was produced by all the lecithinase-positive strains of Bacillus cereus, B. cereus var. mycoides and the crystal-formers. Steinhaus (1951) and Heimpel \& Angus (1958) reported that the two varieties of B. entomocidus did not produce acetylmethylcarbinol and Dr Ellen Garvie (personal communication) has found the same for $\boldsymbol{B}$. cereus 634 . These negative reactions were confirmed. However, the lecithinase negative $B$. cereus var. mycoides strain A.C., B. cereus strains 826 and 827 and crystal-formers $\mathrm{G} 1$ and $\mathrm{G} 2$ were all strongly positive.

\section{Lecithinase activities}

The majority of strains of Bacillus cereus, B. cereus var. mycoides and of the crystal-formers were strong lecithinase producers, the zones of turbidity spreading well beyond the margins of colonies on egg-yolk and lecithin agars. B. cereus 634 and $826, B$. cereus var. mycoides A.C., the two varieties of $\boldsymbol{B}$. entomocidus and crystal-former $\mathrm{G} 2$ were negative in the lecithinase test. Feeble lecithinase production leading to a weak zone of turbidity below the colony and scarcely extending beyond its margin was detected with $B$. cereus 827 and with crystal-former G1.

\section{Phage sensitivities}

None of the strongly lecithinase-positive organisms (Bacillus cereus, B. cereus var. mycoides or crystal-formers) was susceptible to any of the four phages. The sensitivity patterns of the other strains are summarized in Table 1, which also shows the results of tests for the production of lecithinase and acetylmethylcarbinol and certain sugar fermentations, since these characters are used by Heimpel \& Angus (1958) in the classification of the group. Table 2 shows the plating efficiencies of the phages when tested against the different strains as compared with a standard figure of 100 plaque-forming units for the reaction between each phage and the organism on which it was grown. 
Table 1. Phage sensitivities and other characteristics of Bacillus strains

\begin{tabular}{|c|c|c|c|c|c|c|c|c|c|c|c|c|}
\hline \multirow[b]{3}{*}{ Organism } & & & & & \multirow{3}{*}{ 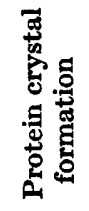 } & \multirow{3}{*}{ 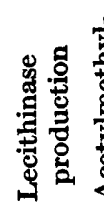 } & \multirow{3}{*}{ 递 } & \multicolumn{5}{|c|}{ Production of acid from } \\
\hline & \multicolumn{4}{|c|}{ Sensitivity to phage } & & & & $\varpi_{0}$ & 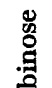 & छั & 兽 & 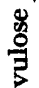 \\
\hline & A & B & C & D & & & & $\hat{x}$ & 4 & ㄲํㄴ & & \\
\hline $\begin{array}{l}\text { B. entomocidus } \\
\text { var. entomocidus }\end{array}$ & + & + & + & + & + & - & - & - & - & + & + & + \\
\hline $\begin{array}{l}\text { B. entomocidus } \\
\text { var. subtoxicus }\end{array}$ & + & + & + & + & + & - & - & - & - & $\mathbf{w}$ & $\mathbf{w}$ & - \\
\hline G1 & - & - & + & - & + & $\mathbf{w}$ & + & - & - & + & + & $\mathbf{w}$ \\
\hline G2 & + & - & - & - & + & - & + & - & - & + & + & + \\
\hline B. cereus 634 & + & + & + & + & - & - & - & - & - & $\mathbf{w}$ & $\mathbf{w}$ & - \\
\hline B. cereus 826 & + & + & + & + & - & - & + & - & - & + & + & + \\
\hline B. cereus 827 & - & - & - & - & - & $\mathbf{w}$ & + & - & - & + & + & + \\
\hline $\begin{array}{l}\text { B. cereus var. } \\
\text { mycoides A.C. }\end{array}$ & - & + & + & + & - & - & + & - & - & + & + & + \\
\hline
\end{tabular}

Table 2. Plating efficiencies of phages $A, B, C$ and $D$ on the susceptible Bacillus strains

\begin{tabular}{|c|c|c|c|}
\hline \multicolumn{4}{|c|}{ Phage } \\
\hline $\mathbf{A}$ & $\mathbf{B}$ & $\mathbf{C}$ & $\mathbf{D}$ \\
\hline \multicolumn{4}{|c|}{ Plating efficiency } \\
\hline 100 & $\mathbf{5 0}$ & 150 & 200 \\
\hline 500 & 100 & 180 & 200 \\
\hline o & $\mathbf{0}$ & 100 & o \\
\hline $\mathbf{5}$ & $\mathbf{0}$ & $\mathbf{0}$ & $\mathbf{0}$ \\
\hline 100 & 5 & 1 & 1 \\
\hline 100 & $\mathbf{5}$ & 100 & 100 \\
\hline $\mathbf{0}$ & 0.5 & $0 \cdot 1$ & 0.3 \\
\hline
\end{tabular}

\section{Plaque morphologies}

Plaque morphology showed considerable variation which was clearly dependent on both the phage and the test organism. Plaques ranged from completely clear areas about $1 \mathrm{~mm}$. in diameter (e.g. phage $\mathbf{A}$ acting on Bacillus entomocidus var. entomocidus; phage $\mathbf{B}$ acting on the variety subtoxicus) to faintly discernible plaques in which there was no complete clearing of the lawn (e.g. most of the plaques formed on $B$. cereus 634 and on $B$. cereus var. mycoides A.C.). In general, phage $\mathbf{D}$ tended to produce smaller and less well-defined plaques than the others; phage $\mathbf{A}$ behaved characteristically in producing turbid plaques on lawns of $B$. entomocidus var. subtoxicus. The plaque morphologies were not sufficiently characteristic to be of taxonomic value.

\section{Phage resistance}

Colonies of phage-resistant organisms frequently developed in areas of confluent lysis resulting from the action of high phage concentrations. Some of these colonies 
were picked and tested for sensitivity to the different phages and for ability to produce lecithinase, acetylmethylcarbinol and protein crystals. In some cases organisms resistant to one phage were treated with a second phage and doubly resistant variants isolated.

Resistance to phage B was frequently accompanied by the development of resistance to phages $\mathbf{C}$ and $\mathbf{D}$ but was not associated with resistance to phage $\mathbf{A}$ except in the case of Bacillus entomocidus var. subtoxicus. Selection for resistance to phage A did not normally yield strains resistant to the other phages, although results were not always clear cut and increased resistance was occasionally seen with several strains. It was possible to obtain organisms showing a wide variety of phage sensitivity patterns and by selection in several steps to derive strains completely resistant to the four phages from originally susceptible strains.

In all cases changed susceptibility to the phages had no effect on the abilities of the organisms to produce lecithinase or acetylmethylcarbinol. Neither were there any instances of gain or loss of ability to form crystals, but strains of $\boldsymbol{B}$. entomocidus var. subtoxicus, which normally produce rather slender delicate bi-pyramidal crystals, produced larger fatter bi-pyramids when they developed resistance to phage $\mathbf{B}$.

\section{DISCUSSION}

Apart from the detailed study of phages derived from Bacillus cereus strain W reported by McCloy $(1951,1958)$ and a brief note by Földes, Meretey \& Varga (1961) our knowledge of phages in the B. cereus group is scant. Only one short communication concerns phages of the crystal-formers. Gochnauer (1960) isolated phages from lysogenic strains which were active against $B$. entomocidus var. entomocidus and against the 'terminalis' strain of $\boldsymbol{B}$. cereus but he made no mention of lecithinase production by his strains. The 'terminalis' strain in my collection is strongly lecithinase positive and resistant to all four of my phages.

Many strains of Bacillus cereus carry temperate phage and mutation of such phage to a form capable of attacking the lysogenic host appears to be common so that spontaneous phage lysis is frequently seen in bacteria of this group. The most thorough investigation of this type of behaviour is that of McCloy $(1951,1958)$ who showed that a strain of $B$. cereus (strain $W$ ) regularly produced a phage $(W \alpha)$ which formed plaques on strain $W$. Strain $W$ was lysogenic with a temperate phage, $\mathbf{W} \beta$, of which $\mathbf{W} \alpha$ was a virulent mutant. A further non-lysogenizing phage, $\mathbf{W} \gamma$, was also found in cultures of strain $W$. The three phages were identical in serological and other characters and McCloy (1958) studied the close relationship which exists between them. In the present work cultures of $B$. entomocidus var. entomocidus and of $B$. cereus 826 behaved like McCloy's strain W in that they regularly produced phages (A and D, respectively) which were able to produce plaques on the host strain. Spontaneous plaques appeared even when the cultures were derived from single spores and these bacteria presumably carry temperate phages and are susceptible to virulent variants of them.

Studies of Bacillus cereus phages show patterns of group specificity at subspecies level. Földes et al. (1961) isolated phages active against the streptomycin-resistant $B$. cereus strain 569 from soil and found them to be active only against certain $B$. cereus strains. The phages did not attack $B$. anthracis or strains of $B$. cereus which 
produced the 'anthrax wall polysaccharide'. Autolysates of phage-resistant strains gave a precipitation reaction with anti-anthrax polysaccharide sera while autolysates of sensitive strains did not give this reaction. The $\boldsymbol{B}$. cereus cell wall polysaccharide which precipitates anthrax antibody consists of galactose and glucosamine; the corresponding polysaccharide of phage-sensitive strains contains glucose, glucosamine and an unknown hexosamine. The phages described by McCloy (1951) attacked certain $B$. cereus strains and the anthrax bacillus. The group of phage susceptible organisms described in the present communication consists of strains with little or no ability to produce lecithinase and includes both crystal-forming and non-crystal-forming bacteria.

Heimpel \& Angus (1958) suggested a scheme of classification of crystal-formers, recognizing parasporal body formation as an essential criterion for separating them from Bacillus cereus and $B$. anthracis and then further subdividing them into $\boldsymbol{B}$. thuringiensis and $\boldsymbol{B}$. entomocidus on a basis of acetylmethylcarbinol and lecithinase production. B. finitimus constitutes a third species in which the parasporal body does not separate from the spore at maturity. It produces both lecithinase and acetylmethylcarbinol and was not susceptible to any of my phages. The Heimpel $\&$ Angus scheme of classification is of considerable value since it brings order into the group but it does not accommodate lecithinase-negative strains of $B$. cereus (which vary in their abilities to produce acetylmethylcarbinol) or lecithinasenegative acetylmethylcarbinol-producing organisms such as B. cereus var. mycoides A.C. and crystal-former G2. It is generally accepted that the crystal-formers are very similar to $\boldsymbol{B}$. cereus. I have observed a common spore precipitinogen present in all the crystal-formers and $\boldsymbol{B}$. cereus strains in my collection. The close relationship between crystal-formers and non-crystal-formers is further supported by the present findings in which phage sensitivity cuts across several of the characters used for subdivision in these organisms.

There seem to be two types of 'Bacillus cereus': one group of strains which produces large amounts of lecithinase and another group which does not do so. Both groups contain strains with the typical rhizoid colonial morphology of the mycoides variety and both groups contain crystal-forming strains, many of which are pathogenic for lepidopterous larvae. The characters discussed above could be used as the basis for a scheme of classification of these organisms. But it is clear that our knowledge of the group is still fragmentary; the present observations may perhaps serve better as a stimulus to further work than as the basis for a taxonomic concept of doubtful validity.

Much remains to be learned about the ecology of crystal-formers. Toumanoff (1960) showed that the disease 'flacherie' caused by a crystal-former in the silkmoth Bombyx mori can be transmitted from one generation of larvae to the next. My own closed colony of Galleria mellonella is normally free from visible disease but crystalforming bacteria (strains G1, G 2) have been isolated from diseased larvae on occasions separated by a year during which there were no signs of disease. Further isolations of organism $\mathbf{G} 2$ have been made from excreta of the colony but infected larvae have remained rare. Such observations emphasize the complexity of the host/parasite relationship. The finding that the two organisms G1 and G2 have a different pattern of phage susceptibility suggests that phage typing might play an important part in its elucidation. 
I am grateful to the Agricultural Research Council for a grant in aid of this work and to Miss Maureen Flanagan for valuable technical assistance. I should like to thank Drs T. Angus, Ellen Garvie, C. L. Hannay, P. C. Fitz-James and S. E. Jacobs for supplying strains of Bacillus cereus and crystal-formers.

\section{REFERENCES}

Adams, M. H. (1959). Bacteriophages. New York: Interscience Publishers Inc.

ANGUs, T. A. (1954). A bacterial toxin paralysing silkworm larvae. Nature, Lond. 173, 545.

Földes, J., Meretey, K. \& VArga, I. (1961). Isolation and some properties of the phages of $B$. cereus NRRL 569. Nature, Lond. 189, 161.

GochnaUer, T. A. (1960). Some bacteriophages from crystalliferous Bacillus spp. pathogenic for certain insects. Bact. Proc. G92.

Hannay, C. L. (1953). Crystalline inclusions in aerobic spore-forming bacteria. Nature, Lond. 172, 1004.

Heimpel, A. M. \& Angus, T. A. (1958). The taxonomy of insect pathogens related to Bacillus cereus Fr. and Fr. Canad. J. Microbiol. 4, 531.

Le Conroller, Y. (1958). A propos de la transformation de souches banales de B. cereus Frank. et Frank. en souches cristallophores pathogènes pour les insectes. Ann. Inst. Pasteur, 94, 670.

McCloy, E. W. (1951). Studies on a lysogenic Bacillus strain. 1. A bacteriophage specific for Bacillus anthracis. J. Hyg., Camb. 49, 114.

McCloy, E. W. (1958). Lysogenicity and immunity to Bacillus phage W. J. gen. Microbiol. $18,198$.

Nungester, W. J. (1929). Dissociation of Bacillus anthracis. J. infect. Dis. 44, 73.

Smith, N. R., Gordon, R. E. \& Clark, F. E. (1952). Aerobic Sporeforming Bacteria. U.S. Dept. Agric. Monograph No. 16.

Steinhaus, E. A. (1951). Possible use of Bacillus thuringiensis Berliner as an aid in the biological control of the alfalfa caterpillar. Hilgardia, $20,359$.

Stone, M. J. (1952). The action of the lecithinase of Bacillus cereus on the globule membrane of milk fat. J. Dairy Res. 19, 311.

Toumanoff, C. (1956). Virulance expérimentale d'une souche banale de Bacillus cereus Frank. et Frank. pour les chenilles de Galleria melonella L. et Pieris brassicae. Ann. Inst. Pasteur, 90, 660.

Toumanoff, C. (1960). Observations sur la transmission héréditaire de la flacherie des vers-à-soie (Bombyx mori L.). Ann. Inst. Pasteur, 98, 367.

Willis, A. T. (1960). The lipolytic activity of some clostridia. J. Path. Bact. 80, 379. 